\section{Pulssynchroner Tinnitus als einziges Symptom einer fibromuskulären Dysplasie der zervikalen Arterien}

Ohrgeräusche gehören zu den häufigsten Beschwerden, die Patienten zum HNOArzt führen. Das pulsatile Ohrgeräusch ist dagegen relativ selten. Es entsteht häufig durch Turbulenzen im normalerweise laminären Blutstrom bei absoluten oder relativen Stenosen im Gefäßlumen. Die Ursachen des pulsatilen Tinnitus sind vielfältig. Häufig ist die Diagnose schwierig und bei vielen Patienten mit zahlreichen frustranen Konsultationen verschiedener Fachärzte verbunden. In Frage kommen vaskuläre Erkrankungen, erhöhtes Herzminutenvolumen bei Hyperthyreose oder Anämie, erhöhter intrakranieller Druck und gefäßreiche Tumoren. Bei den arteriellen Veränderungen kommen als Ursachen Aneurysmen der A.carotis interna $(\mathrm{ACI})$, eine lateropositionierte oder im Mittelohr freiliegende ACI, arterielle Gefäßanomalien im Kleinhirnbrückenwinkel und stenosierende arteriosklerotische Plaques vor. Im venösen Schenkel des Blutkreislaufes ist ein hochstehender Bulbus venae jugularis bei enger Nachbarschaft zum Mittelohr manchmal Ursache des pulssynchronen Ohrgeräusches (Mahlo HW et al., Laryngo-Rhino-Otol 1991, 70: 675-677). Selten kann auch die Fibromuskuläre Dysplasie (FMD) der ACI (Waldvogel D et al., J Neurol 1998; 245: 137 -142) oder der A. vertebralis (AV) als Ursache eines pulssynchronen Tinnitus nachgewiesen werden. Die Diagnose in unserem Fallbeispiel stützt sich auf angiographische Kriterien, eine histologische Klassifizierung liegt bislang nicht vor.

\section{Fallbericht}

Die 52jährige Patientin kam aufgrund eines seit $1 \frac{1}{2}$ Jahren bestehenden pulssynchronen Tinnitus rechts zur stationären Aufnahme in die HNO-Klinik. Bei der klinischen Untersuchung bestand eine etwa $1,5 \mathrm{~cm}$ große pulsierende Raumforderung im linken Kieferwinkel. Diese hatte die Patientin erstmalig vor etwa 2 Jahren bemerkt. Darüber hinaus waren die allgemeinmedizinische Untersuchung, die weitere HNO-Diagnostik (Audiogramm, Spiegelbefund) und die neurologische Untersuchung unauffällig. Anamnestisch und aktuell lag keine arterielle Hypertonie vor. In der auswärtigen Computertomographie war eine unklare KM-Anreicherung im Kieferwinkel links beschrieben worden. Zum Ausschluß eines Glomustumors wurde eine i. a. DSA der Karotiden durchgeführt. Statt eines gefäßreichen Tumors fanden sich jedoch beidseitig multiple kurzstreckige, konzentrische Stenosen der distalen extrakraniellen ACI-Segmente bei jeweils unauffälliger Darstel-
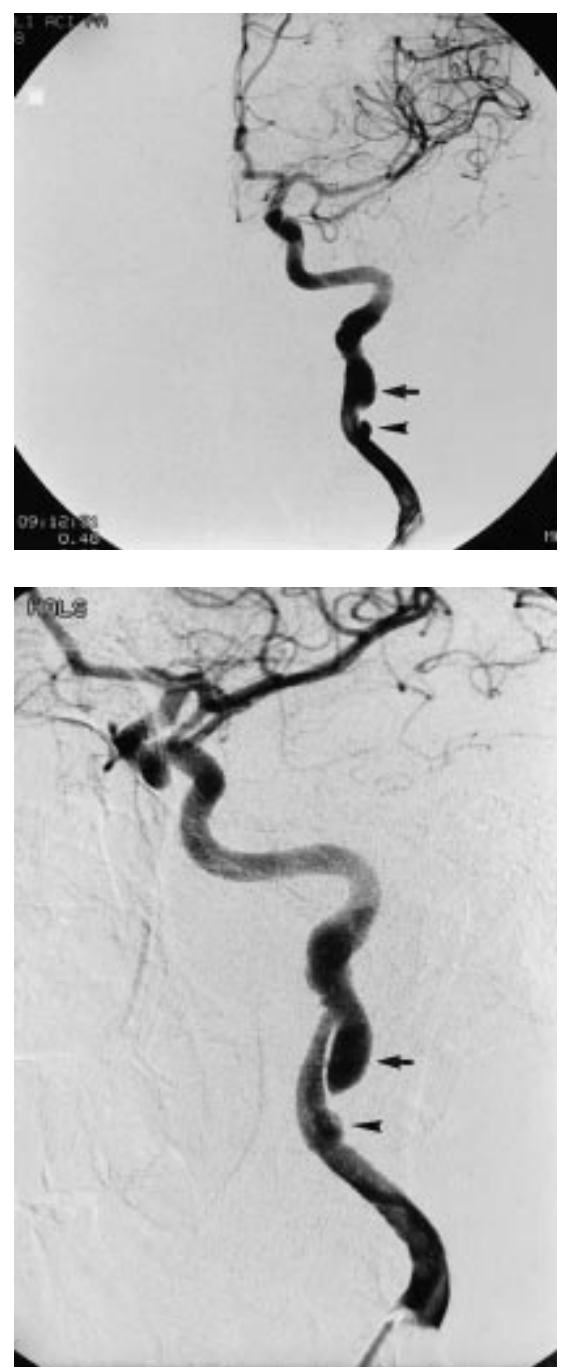

Abb. 1 Großes, sackförmiges (Pfeil) und kleineres, kaudal gelegenes (Pfeilspitze) Pseudoaneurysma der linken A. carotis intema in AP(a) und LAO- (b) Projektion. Dazwischenliegend stenosierter Abschnitt nach mutmaßlicher spontaner Dissektion. lung der proximalen und auch der intrakraniellen Abschnitte (insbesondere ohne Nachweis von intrakraniellen Aneurysmen). Ferner konnten an der linken ACI zwei aneurysmatische Erweiterungen des Lumens und eine langstreckige mäßiggradige Stenose des dazwischenliegenden Gefäßabschnittes (Abb.1au.b), dargestellt werden. Das distale größere Aneurysma im subpetrösen Abschnitt der ACI entsprach der Lokalisation des tastbaren Tumors. Bei der nun geänderten Verdachtsdiagnose wurden eine Untersuchung der AV bds. und eine Übersichtsserie der Nierenarterien im gleichen Untersuchungsgang angeschlossen. Sowohl an den Vertebralarterien (Abb. 2au.b) als auch an den Nierenarterien (Abb. 3) konnten gleichartige Gefäßveränderungen nachgewiea sen werden. Nach angiographischen Kriterien (Osborn AG et al., Stroke 1977; 5: 617-626) wurde die Diagnose einer FMD der Hals- und Nierenarterien mit Z.n. spontaner, asymptomatischer ACI-

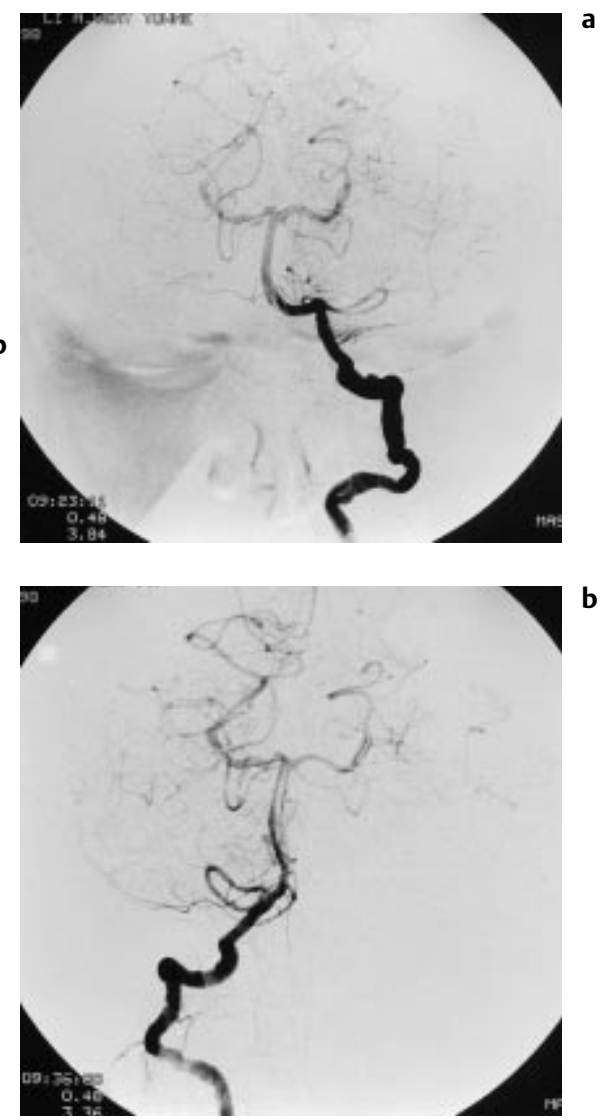

Abb. 2 Perlschnurartig aneinandergereihte Stenosen alternierend mit Lumenerweiterungen der distalen extrakraniellen A. vertebralis links (a) und rechts (b) (sog. „string of beads“Sign). 


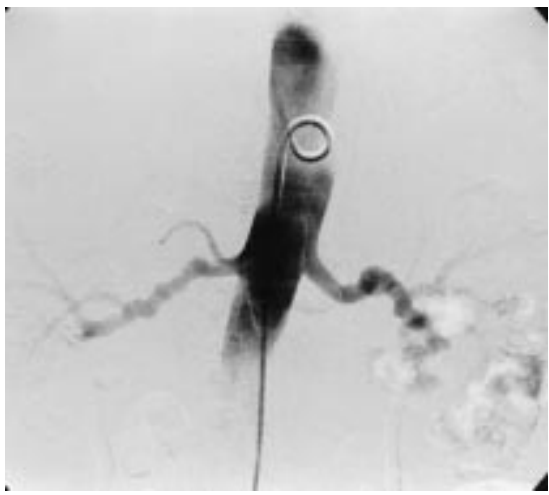

Abb. 3 FMD-Befall auch der Nierenarterien mit typischem string of beads-sign.

Dissektion und Bildung von Pseudoaneurysmen links gestellt. Es wurde eine Antikoagulation der Patientin eingeleitet, um thrombembolische Komplikationen zu verhindern.

Zur weiteren Abklärung der Genese des Tinnitus wurden neben einem Kompressionsversuch an der rechten A.carotis communis auch selektive Kompressionen der AV und der A.occipitalis unter dopplersonographischer Kontrolle durchgeführt. Das Ohrgeräusch sistierte bei Kompression der rechten $A$. vertebralis.

\section{Diskussion}

Die Fibromuskuläre Dysplasie ist eine seltene, ätiologisch unklare stenosierende Erkrankung der Arterien, die überwiegend Frauen im mittleren Lebensalter befällt. Histologisch ist die FMD charakterisiert durch Proliferation von fibrösem Gewebe, Hyperplasie der glatten Muskelzellen einerseits und alternierend Zerstörung von elastischen Fasern in der arteriellen Gefäßwand mit konsekutiven Wandverdünnungen (Harrison EG et al., Mayo Clin Proc 1971; 46: 161 167).

Bei der FMD der zervikokranialen Arterien führen diese hyperplastischen Läsionen angiographisch zu verschiedenen Erscheinungsbildern: Am häufigsten (ca. 85\% der Fälle) finden sich alternierende Stenosen des Gefäßlumens, die ein perlschnurartiges Erscheinungsbild („string of beads“) der betroffenen Gefäße hervorrufen. Weniger häufig und weniger pathognomonisch finden sich langstrekkige tubuläre Stenosen. Durch den Abbau von elastischen Fasern kann es zu spontanen Dissektionen und Pseudoaneurys- men der Gefäßwände, zur sogenannten „atypischen“ FMD, kommen. Bei einem Befall intrakranieller Gefäße kann es zur Ausbildung von multiplen Aneurysmen kommen (Mettinger KL et al., Stroke 1982; 13: 46-52).

An den Halsarterien manifestiert sich die FMD meist an den distalen, aber extrakraniellen Abschnitten der Karotiden und der AV, oft auf Höhe des ersten und zweiten Halswirbels. Die Karotisbifurkation und das proximale AV-Segment bleiben ausgespart.

Klinisch macht sich die FMD der Halsarterien meist durch transitorische ischämische Attacken (TIAs), zerebrale Infarkte oder, bei intrakraniellem Befall, durch eine subarachnoidale Blutung (SAB) bemerkbar. Tinnitus ist mitunter als Begleitsymptom bei rezidivierenden TIAs und Schwindel beschrieben worden. Berichte über Tinnitus als einziges Symptom der FMD (in unserem Fall hervorgerufen durch turbulente Strömungen in der rechten AV) konnten wir in der Literatur nicht finden. Da in unserem Fall alle drei der von Osborn beschriebenen Typen der FMD zu finden sind, kann mit großer diagnostischer Sicherheit auch ohne histologischen Beleg die Diagnose allein angiographisch gestellt werden.
Therapeutisch stehen zur Behandlung der Stenosen und Aneurysmen der zervikalen FMD prinzipiell neben der Operation die endovaskulären Interventionsoptionen Angioplastie, Stenting, Coiling oder die Kombination Stenting und Coiling bei Pseudoaneurysmen der $\mathrm{ACI}$ zur Verfügung. Anders als bei traumatischen Pseudoaneurysmen der ACI bleibt aber aufgrund der generellen Pathologie der Gefäßwand bei der FMD die operative oder endovaskuläre Intervention lediglich komplizierten Einzelfällen vorbehalten. Bei asymptomatischen Patienten kann die Therapie auf eine Antikoagulation zur Vorbeugung thrombembolischer Komplikationen beschränkt bleiben, denn bei der FMD besteht im spontanen Verlauf bei ACIStenosen durch Dissektionen eine Tendenz zur Normalisierung des Gefäßlumens (Manninen HI et al., AJNR 1997, 18: $1216-1220)$.

Obwohl meist blande verlaufend, können die zerebrovaskulären Komplikationen der Erkrankung in Form von Ruptur intrakranieller Aneurysmen und thrombembolischen Hirninfarkten lebensbedrohend sein. Deshalb ist die Fibromuskuläre Dysplasie der kraniozervikalen Arterien bei der Abklärung von Tinnitus in die Differentialdiagnose einzubeziehen.

C. Weihl, A. Dörfler, M. Forsting, Essen

\section{Intermittierende Invagination beim Peutz- Jeghers-Syndrom: CT- und MR-Befunde}

Das Peutz-Jeghers-Syndrom (PJS), erstmals beschrieben 1929 von Peutz, später von Jeghers, ist eine seltene Krankheit mit unregelmäßig geformten mukokutanen Pigmentflecken und neuromuskulären Darmpolypen. Das Vererbungsmuster ist autosomal-dominant. Kürzlich wurde der verantwortliche Genlocus auf Chromosom 19p isoliert. Die Ausprägung der Darmpolypose bestimmt das Beschwerdebild der Patienten mit Neigung zu gastrointestinalen Blutungen, Abdominalschmerzen durch intermittierende Invaginationen und Pankreatitiden. Eine mögliche karzinomatöse Entartung der Polypen sowie ein erhöhtes Risiko extraintestinaler Malignome ist mit der Erkrankung assoziiert. Im folgenden wird über eine
Patientin mit ausgeprägter Polyposis und intermittierender Invagination berichtet, und die compu-tertomographischen und magnetresonanztomographischen Befunde werden dargestellt.

\section{Fallbericht}

Eine 42jährige Patientin stellte sich mit starken krampfartigen Oberbauchschmerzen vor, die seit zwei Tagen progredient waren. Innerhalb der letzten zwei Jahre hatte die Patientin intermittierend über ähnliche rezidivierende epigastrische Schmerzen nach Nahrungsaufnahme geklagt, die sich jeweils ohne Therapie zurückbildeten. Vor 10 Monaten war ein positiver Hämoccult-Test abgenommen und eine Eisenmangelan- 


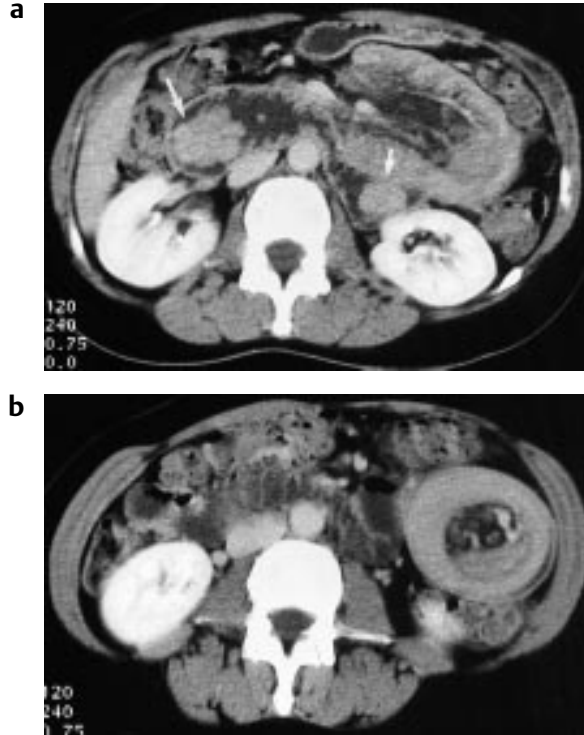

Abb.1 (a) Computertomographie: Intestinale Polypose erkennbar an einem $4 \mathrm{~cm}$ großen Polyp im flüssigkeitsgefüllten Duodenum (langer Pfeil) und einem $2,5 \mathrm{~cm}$ großen Polyp im proximalen Jejunum (kurzer Pfeil). Partiell miterfaßte Invagination im linken Hemiabdomen. (b) Computertomographie: Typische kokardenförmige Darm-in-Darm-Erscheinung einer Dünndarminvagination im linken Hemiabdomen.

ämie diagnostiziert worden. Zu gleicher Zeit war aufgrund auffälliger perioraler Pigmentflecken erstmals der Verdacht auf ein PJS geäußert worden und an Gewebsproben aus Magenpolypen die Diagnose gesichert worden. Die 4 Töchter der Patientin wiesen die gleichen perioralen Pigmentierungen auf.

Zur initialen Abklärung der Schmerzsymptomatik wurde eine Computertomographie des Abdomens (Somatom Plus 4, Siemens, Erlangen; $5 \mathrm{~mm}$ Kollimation, $8 \mathrm{~mm}$ Tischvorschub, $4 \mathrm{~mm}$ Inkrement) nach oraler und intravenöser KMApplikation $(150 \mathrm{ml}$ Imeron $300 \mathrm{mg} / \mathrm{ml}$, Fluß $3 \mathrm{ml} / \mathrm{s}$ ) durchgeführt, die multiple intraluminäre Raumforderungen in den Dünndarmschlingen des Duodenums und proximalen Jejunums zeigte (Abb.1). Zusätzlich fand sich eine kokardenartige Raumforderung im linken $\mathrm{Ab}$ domen (Abb. 2). Eine Darm-in-DarmErscheinung und eingeschlossene Mesenterialgefäße ließen auf eine Invagination schließen. Differentialdiagnostisch war unter Berücksichtigung der intestinalen Blutungen auch an eine intramurale Einblutung zu denken. Zur Differenzierung wurde daher am darauf-
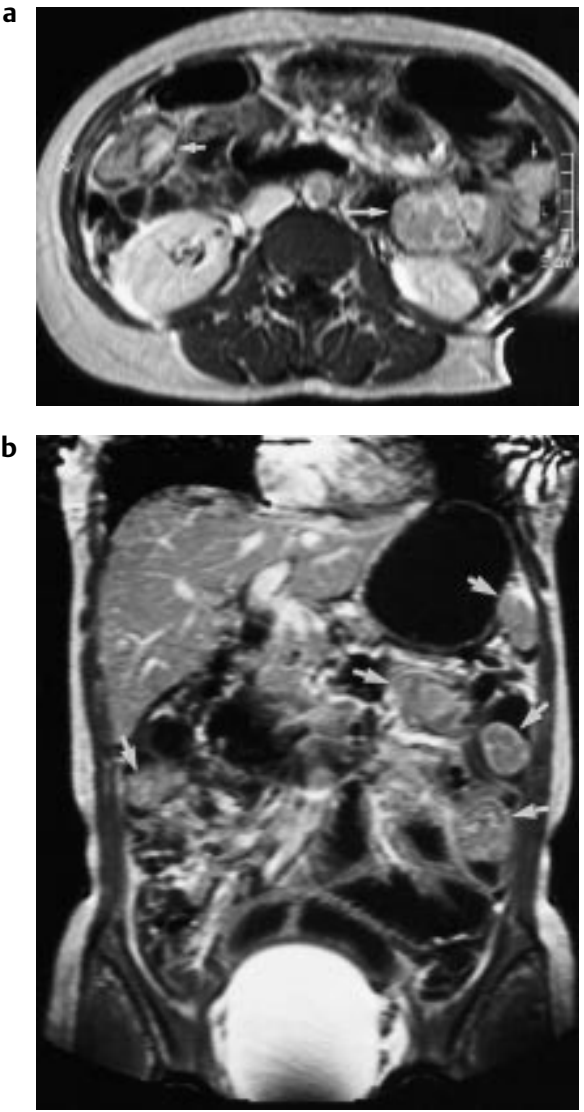

Abb. 2 (a) Magnetresonanztomographie, transversale Schnittführung: $4,5 \mathrm{~cm}$ großer multilobulierter Polyp im proximalen Jejunum (langer Pfeil), 2,5 cm großer Polyp in einer Dünndarmschlinge im linken Hemiabdomen (kleiner Pfeil) sowie $2,5 \mathrm{~cm}$ großer Polyp im rechten Hemiabdomen

(kurzer Pfeil) ( $\mathrm{T}_{1}$-gewichtete FFE-Sequenz nach i.v. KM-Gabe). (b) Magnet-

resonanztomographie, koronale Schnittführung: Multiple intraluminäre, KM-

aufnehmende Raumforderungen im proximalen Dünndarm entsprechend hamartomatösen Polypen bei PJS (gekennzeichnet).

folgenden Tag eine Magnetresonanztomographie (Philips, Best, NL, Gyroscan ACS NT, 1,5 Tesla) durchgeführt. Nach Applikation von 1,5 Liter Methylcellulose oral wurden $\mathrm{T}_{2}$-gewichtete TSE-Sequenzen in axialer und koronarer Schnittführung (TR: $2350 \mathrm{~ms}$, TE: $100 \mathrm{~ms}$ ) sowie $\mathrm{T}_{1}$ gewichtete Gradienten-Echo-Sequenzen (TR: $184 \mathrm{~ms}$; TE: 6,9 ms; Flipwinkel: 60 ${ }^{\circ}$ ) vor und nach i.v. Kontrastmittelgabe (Gd-DTPA, Magnevist ${ }^{\circledR}$ ) durchgeführt. Zur Reduktion der Darmperistaltik wurden $40 \mathrm{mg}$ Buscopan vor der Untersuchung i. v. verabreicht. Die Darmpolypen waren als intraluminäre, überwiegend polyglobulierte Raumforderungen von $2 \mathrm{~mm}-5 \mathrm{~cm}$ Größe deutlich identifizier- bar. In $\mathrm{T}_{1}$ - und in $\mathrm{T}_{2}$-Wichtung erschienen die Polypen intermedär signalintens und wiesen ein deutliches KM- Enhancement auf. Die kokardenförmige Raumforderung im linken Hemiabdomen war in der Magnetresonanz-tomographie nicht mehr nachweisbar, für eine intramurale Einblutung gab es keine Hinweise. Die CT-Diagnose einer Invagination, die sich zwischenzeitlich wieder zurückgebildet hatte, wurde somit unterstützt.

\section{Diskussion}

Das Peutz-Jeghers-Syndrom ist eine seltene Erkrankung, charakterisiert durch mukokutane Pigmentierungen und hamartomatöse Polypen im GI-Trakt. Trotz der typischen mukokutanen Pigmentflecken bei Kindern und Jugendlichen wird die Diagnose eines PJS - wie in dem hier beschriebenen Fall - häufig erst durch den Nachweis der Darmpolypen gestellt. Dieser gelingt üblicherweise mit einem Barium-Enteroklysma. Die Polypen erscheinen hier als Füllungsdefekte und können unterschiedliche Anzahl, Größe und Form aufweisen. In absteigender Häufigkeit sind Jejunum, Duodenum, Ileum, Kolon und Magen betroffen (Cho et al., Radiographics 1997; 17: 785 791). Wegen der großen Variabilität der Größe, Form und Lokalisation der Polypen ist eine sichere Unterscheidung von anderen Polypose-Syndromen nicht möglich. Daher müssen differentialdiagnostisch ähnliche Erkrankungen wie die Familiäre Adenomatöse Polyposis Coli (FAPC), die tuberöse Sklerose, das Cronkhite-Canada Syndrom und andere erwogen werden.

Während das Enteroklysma die sensitivste Untersuchung zum Nachweis der Polypen ist, eignen sich Ultraschall und CT zum Nachweis von Invaginationen. In beiden Untersuchungsverfahren ist eine Invagination an der typischen Kokardenform der Darm-in-Darm-Erscheinung zu erkennen. Differentialdiagnostisch wurde in unserem Fall neben einer Invagination auch an eine Darmwandeinblutung gedacht.

Spontane intramurale Dünndarmblutungen sind in den letzten Jahren häufiger berichtet worden und oft klinisch nicht zu vermuten (Lane et al., Clin Radiol 1997; 52: 378-80).

Die Abklärung führten wir mittels MRT durch. Die MRT hat in der Diagnostik 
entzündlicher Darmerkrankungen zunehmende Bedeutung gewonnen. Dagegen liegen über die Diagnostik von Dünndarmtumoren mit der Magnetresonanztomographie nur unzureichende Erfahrungen vor. Die Darstellung eines PJS in der MRT ist in der Literatur bislang nur in einem Fall beschrieben. Bei diesem Patienten konnte eine Intussusception MR-tomographisch dargestellt werden (Marcos et al., Magn Res Imaging 1997; 15: 1095-1098). Theoretische Vorteile der MRT gegenüber der CT ergeben sich durch den hohen Weichteilkontrast und die fehlende Strahlenbelastung. Da es sich bei PJS-Patienten häufig um jüngere Patienten handelt, kommt letzterem besonderes Gewicht zu.

Um eine homogene Kontrastierung und gute Distension der Dünndarmschlingen $\mathrm{zu}$ erreichen, wurde vor der Untersuchung Methylcellulose (1,5-21) oral und zur Reduktion der Darmperistaltik Buscopan i.v. verabreicht. Diese Methode hatte sich in der MR-Diagnostik entzündlicher Darmerkrankungen bewährt. $\mathrm{T}_{1}$-gewichtete Gradienten-Echo- bzw. Turbo-Spin-Echo-Sequenzen erlaubten eine Untersuchung in Atemanhaltetechnik. Die MRT konnte die Darmpolypen in gleicher Qualität wie die CT nachweisen. Die beste Abgrenzbarkeit der Tumoren war in der $\mathrm{T}_{1}$-gewichteten FFE-Sequenz nach i.v. KM-Applikation gegeben. Die in der CT am Vortag gesehene kokardenförmige Raumforderung war in der MRT nicht mehr nachweisbar, eine Darmwandeinblutung konnte sicher ausgeschlossen werden.

Patienten mit PJS weisen ein erhöhtes Risiko für die Entwicklung extraintestinaler Malignome auf. Die häufigsten dieser Tumoren betreffen Mamma, Pankreas und die Reproduktionsorgane (Giardella et al., N Engl J Med 1987; 316; 1511 - 1514). Außerdem scheint eine mögliche maligne Transformation der Polypen gesichert. Daher ist eine exakte, frühzeitige Diagnostik notwendig und eine Entfernung der Polypen angeraten. Während die Standardtherapie früher in einer Laparotomie mit Polypabtragung oder Darmteilresektion bestand, wird in jüngster Zeit eine möglichst vollständige Entfernung der Polypen bei gleichzeitiger Schonung des Darms mittels Endoskopie empfohlen (Mathus-Vliegen et al., Endoscopy 1995; 17: $102-104)$. In dem hier beschriebenen Fall wurde aufgrund der besonderen Ausprägung der Polypose ein kombiniert chirurgischendoskopischer Eingriff durchgeführt.

David Maintz, Köln

\section{MR-Bildgebung bei Rhabdomyolyse mit Kompartmentsyndrom des Beckens und der Oberschenkel}

Das Kompartmentsyndrom (KS) wurde 1975 von Matsen vereinheitlichend definiert: „A compartmental syndrome is a condition in which increased pressure within a limited space compromises the circulation and the function of the tissues within that space." Als Erstbeschreiber wies Richard von Volkmann bereits 1881 auf die Zusammenhänge einer unterbrochenen Blutversorgung und der Entstehung von Kontrakturen bei der suprakondylären Humerusfraktur hin. Das klassische KS ist für die Sketettmuskulatur beschrieben, wobei das Krankheitsbild heutzutage auch auf andere Organsysteme wie Cerebrum, Auge oder Darm übertragen wird (Echtermeyer et al., Unfallchirurg 1977; 100: 924). Klinisch unterscheidet man zwischen akutem und chronischem sowie zwischen drohendem und manifestem
Seltener sind die Manifestationen am Unterarm, an der Hand, am Fuß oder am Becken. Die Ursachen sind in erster Linie Unfälle mit Frakturen, Weichteilschäden, Gefäßverletzungen und Verbrennungen, aber auch ungewohnte körperliche Belastungen, Einblutungen bei Gerinnungsstörungen oder alkohol- und drogeninduzierte Komata mit lageabhängiger Kompartmentschwellung (Huber et al., Chirurg 1997; 68: 829). Als Circulus vitiosus kann nicht nur das KS eine Rhabdomyolyse verursachen, sondern auch umgekehrt die Rhabdomyolyse ein KS initiieren. Bei der Rhabdomyolyse werden innerhalb von Stunden Zellbestandteile, Metabolite und Entzündungsmediatoren freigesetzt, welche die glomeruläre Filtration vermindern und zum akuten Nierenversagen führen. Die traumabedingte Rhabdomyolyse kombiniert mit Nierenversagen und Schocksymptomatik hat Bywaters 1953 als „crush syndrome“ definiert. Hiervon abzugrenzen sind die nicht traumabedingten myonephropathischen Erkrankungen, die häufig durch eine toxische Rhabdomyolyse ausgelöst werden und in dem gleichen lebensbedrohlichen Circulus vitiosus enden.

\section{Fallbeschreibung}

Ein 30jähriger Patient mit bekannter Heroinanamnese hatte $14 \mathrm{ml}$ L-Polamidon ${ }^{\circledR}$-Tropfen (=60 mg Levomethadon) und 25 Tabletten des trizyklischen Antidepressivums Aponal ${ }^{\circledR} 50 \quad$ (= $1250 \mathrm{mg}$ Doxepin) zu sich genommen und anschließend 12-15 Stunden im Bett schlafend verbracht. Er erwachte mit akuten Schmerzen der Glutealregion. In der Notaufnahme war der Patient ansprechbar, aber nicht vollständig orientiert. Die Untersuchung wies eine Verhärtung der Oberschenkelstreckmuskulatur links nach. Die Muskulatur des Beckens, des rechten Oberschenkels und beider Unterschenkel war palpatorisch unauffällig. Es ließ sich ein Druckschmerz über dem $\mathrm{N}$. ischiadicus rechts provozieren. Motorik und arterielle Durchblutung beider Füße waren normal. Der Patient hatte eine Myoglobinurie, und das Notfall-Labor ergab: Kreatinkinase (CK) $37950 \mathrm{U} / \mathrm{I}(<80 \mathrm{U} / \mathrm{l})$, Myoglobin $75960 \mathrm{mg} / \mathrm{l} \quad(<75 \mathrm{mg} / \mathrm{l})$, Kreatinin 2,2 mg/dl (0,6-1,5 mg/dl) und Kalium 5,4 mmol/l (3,5-5,0 mmol/l). Unter der Verdachtsdiagnose eines KS wurde eine Magnetresonanztomographie (MRT) des Beckens und der Oberschenkel angefertigt.
Die häufigste Lokalisation des Kompartmentsyndroms ist der Unterschenkel mit seinen 4 getrennten Muskellogen. 
a, b

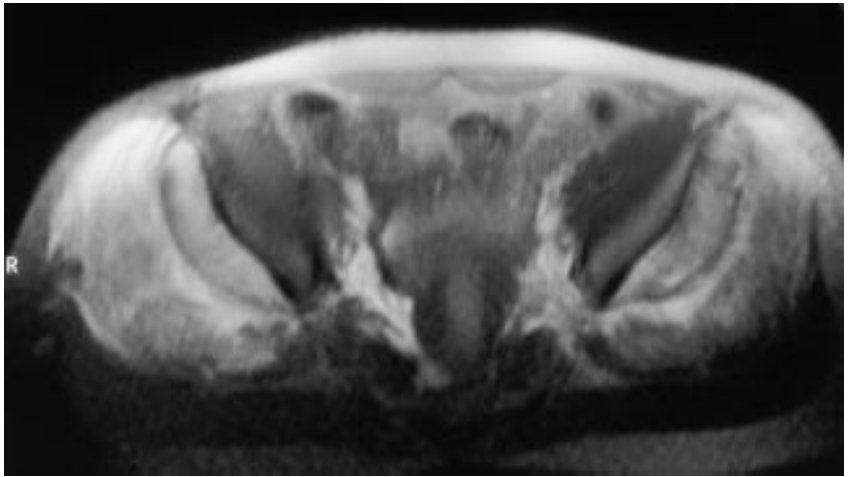

C, d

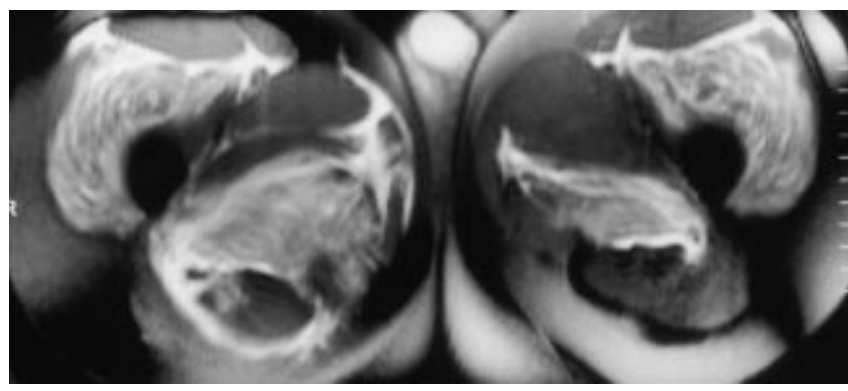

Abb.1a-d Kompartmentsyndrom am Becken und beiden Oberschenkeln. In der TIRM-Sequenz lassen sich ausgedehnte Ödemzonen der Mm. glutaei med. und min. bds. nachweisen (a). Am Oberschenkel sind die Mm. adductor longus, adductor magnus und adductor brevis
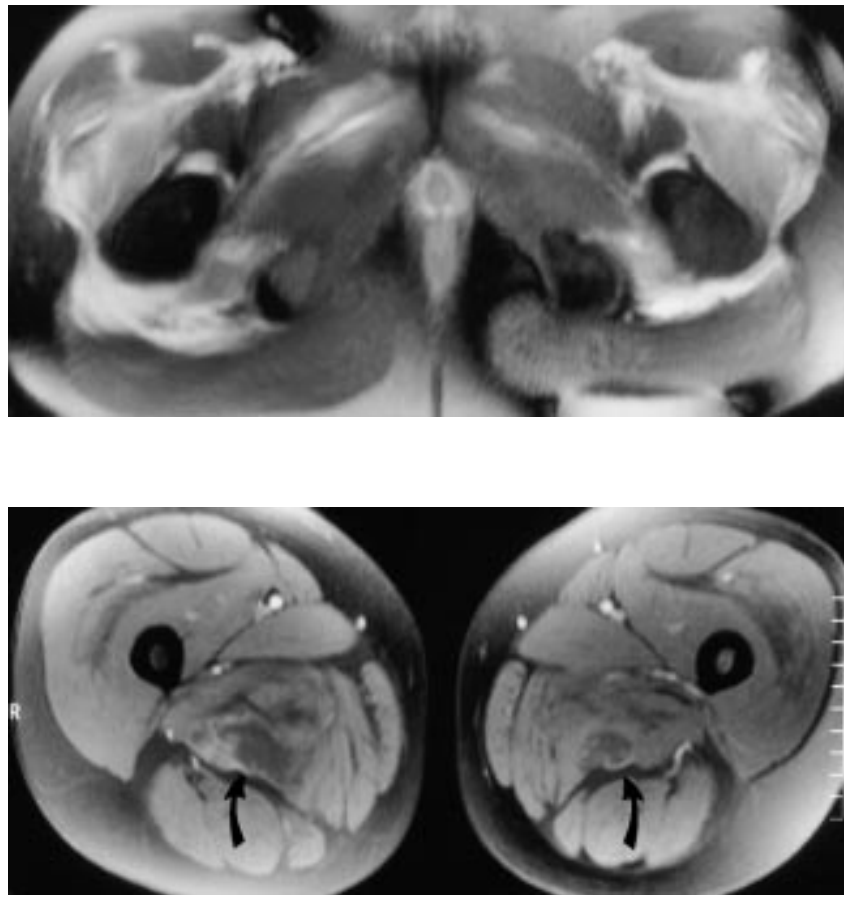

ödematös durchsetzt (b). Von der Streckmuskulatur ist insbesondere der M. vastus lateralis betroffen (c). Nach Kontrastmittelgabe zeigt eine fettgesättigte $T_{1}$-gewichtete Sequenz eine inhomogene Perfusion der Adduktoren (Pfeile in d).
In einer flüssigkeitssensitiven TIRM(turbo-inversion-recovery-measurement) Sequenz wurden ausgedehnte Ödemzonen der Skelettmuskulatur nachgewiesen. Am Becken war insbesondere die gemeinsame Muskelloge der Mm.glutaei medius und minimus beidseits betroffen. Die Mm. glutaei maximus und iliopsoas, die jeweils eine eigene Muskelloge besitzen, waren unauffällig (Abb.1 a). Am Oberschenkel zeigten die $\mathrm{Mm}$. adductor longus, adductor magnus und adductor brevis eine Ödembildung, wobei der Befund rechts erheblich ausgeprägter war als links (Abb. 1 b u. c). Von der Streckmuskulatur waren insbesondere die Mm. vastus lateralis und inter-

\section{„Springende Punkte“}

- Das klassische KS wird klinisch diagnostiziert.

- In fraglichen Fällen sollte eine Schichtbildgebung erfolgen.

Die MRT ist das Verfahren der Wahl. medius auf beiden Seiten betroffen (Abb.1c). Nach Kontrastmittelapplikation ließen sich Areale fehlender KMAufnahme im M. adductor magnus beidseits nachweisen, die nekrotischen Muskelanteilen entsprachen (Abb.1 d).

In der anschließenden Operation bestätigte sich das Bild des KS und die betroffenen Kompartimente wurden fasziotomiert. Nach dem Eingriff fiel die CK um $10000 \mathrm{U} / \mathrm{l}$ ab. Das Kreatinin erreichte aufgrund eines akuten Nierenversagens am 4. postoperativen Tag mit einem Wert von 9,9 mg/dl ein Maximum.

\section{Diskussion}

Die Skelettmuskulatur ist im Gegensatz $\mathrm{zu}$ anderen Organsystemen sehr resistent gegen alle Arten von Schädigungen. Aus diesem Grund tritt die medikamenten-induzierte Rhabdomyolyse sehr selten auf (Titley et al., Intensive Care Med 1992; 18: 129). Eine Schädigung der Kationenpumpe innerhalb der Zellmembran führt $\mathrm{zu}$ einer zunehmenden Schwellung der Zelle mit Ausbildung autolytischer Prozesse. Die Freisetzung größerer Mengen von Myoglobin und Kalium in den Zentralkreislauf löst durch eine Präzipitation des Myoglobins in den renalen Tubulus eine tubuläre Nekrose mit akutem Nierenversagen aus. Um das Abgleiten der Rhabdomyolyse in das lebensbedrohliche myonephropathische Syndrom aufhalten zu können, ist eine sofortige Diagnose und Therapie notwendig. Entscheidend ist hierfür, die betroffenen Muskellogen zu detektieren, um durch eine Fasziotomie das entstehende KS zu entlasten und damit den Circulus vitiosus zu unterbrechen.

Die Symptome des manifesten KS sind krampfartig bohrende Schmerzen, gefolgt von Hypästhesien bis hin zur völligen Gefühllosigkeit. Störungen der Muskelfunktion sind schon nach kurzer Ischiämiezeit nachweisbar. Die subfasziale Gewebedruckmessung bestätigt die klinische Diagnose, was die sofortige Fasziotomie indiziert (Becker et al., Chirurg 1987; 58: 764). Insbesondere in der typischen Lokalisation am Unterschenkel bedarf es keiner zusätzlichen Bildgebung, welche die Therapie nur unnötig verzögern und den Zellschaden vergrößern würde. Gänzlich anders ist die Situation beim beginnenden KS, bei nicht kooperationsfähigen Patienten, oder wenn das KS tief gelegene Muskel- 
logen betrifft, die der subfaszialen Druckmessung nicht zugänglich sind. Hier ist es von entscheidender Bedeutung, das Ausmaß der Erkrankung über ein bildgebendes Verfahren zu detektieren. Die Computertomographie konnte in der Vergangenheit bei dieser Fragestellung nur bedingt hilfreich sein. Heutzutage ist die MRT das Verfahren der Wahl, wenn es darum geht, Muskelödeme und -nekrosen zu detektieren und die Topographie der Schädigung festzulegen (Eskelin et al., Radiology 1998; 206: 333). Bekanntermaßen sind hierfür $\mathrm{T}_{2}$-gewichtete Sequenzen geeignet, wobei insbesondere die TIRM-Sequenz aufgrund ihrer Flüssigkeitssensibilität überlegen ist. Perfusionsstörungen können mittels intravenöser Kontrastmittelapplikation nachgewiesen werden. Beiträge in der Literatur bestätigen die eindeutigen Vorteile dieser Methode gegenüber anderen bildgebenden Verfahren.

J. Rodenwaldt, K. Dresing, E. Grabbe, Göttingen

\section{Computertomographische Diagnostik einer nephro-intestinalen Fistel}

Pathologische Verbindungen zwischen dem Hohlsystem der Niere und dem Gastrointestinaltrakt treten selten auf. In einer Literaturübersicht wurden bis 199073 Fälle beschrieben (Hode et al., J Chir 1990; 127: 281-285), und in den 90er Jahren wurde nur von 4 weiteren Fällen berichtet (Morris et al., J Urol 1991; 146: 835-837; Suhler et al., Ann Urol Paris 1995; 29: 8-10 ; Nayir et al., Pediatr Radiol 1995;25:229-230; Nagatsuma et al., Hinyokika Kiyo 1997; 43: 37 - 39). Typischerweise ist das Nierenbecken Ursprung der Fistel. Die Patienten werden durch Flankenschmerzen, Fieber und Anstieg der Entzündungsparameter klinisch auffällig.

\section{Fallbericht}

Ein 61jähriger Patient wurde wegen einer rechtsseitigen Hemiparese mit motorischer Aphasie in der Neurologischen Klinik aufgenommen. Hier entwikkelte er Temperaturen bis $40^{\circ} \mathrm{C}$. Im Rahmen einer ersten urologischen Untersuchung wurden sonographisch ein verschmälerter Parenchymsaum der rechten Niere und ein extrarenales Nierenbecken festgestellt und die Diagnose einer hydronephrotischen Schrumpfniere mit begleitendem Harnwegsinfekt gestellt. Unter der eingeleiteten Antibiotikatherapie kam es zu einer weitgehenden Entfieberung bei persistierender Leukozytose. Nach 14 Tagen erfolgten ein erneuter Fieberanstieg und eine Verlegung des Patienten in die urologische Abteilung. Bei der körperlichen Untersuchung war das rechte Nierenlager druck- und klopfschmerzhaft. Die Labor- untersuchungen ergaben erhöhte CRP-, Leukozyten- und Amylasewerte. Sonographisch ließ sich nunmehr eine $8 \mathrm{~cm}$ große liquide Raumforderung darstellen,

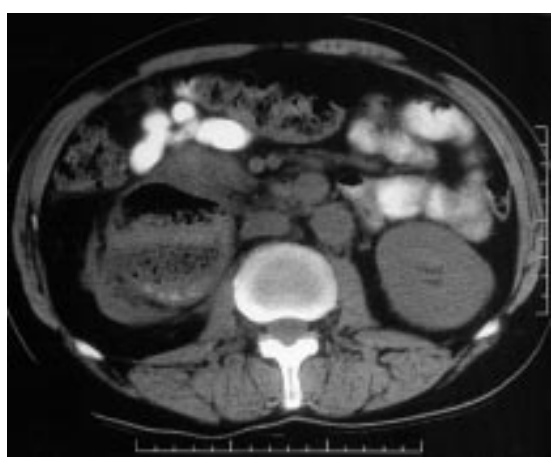

Abb. 1 Nativphase: Nachweis einer $8 \times 8 \mathrm{~cm}$ großen Raumforderung, die vom oberen Nierendrittel ausgeht, bis zum Duodenum reicht und flüssige Anteile sowie einzelne Gasblasen und orales Kontrastmittel enthält.

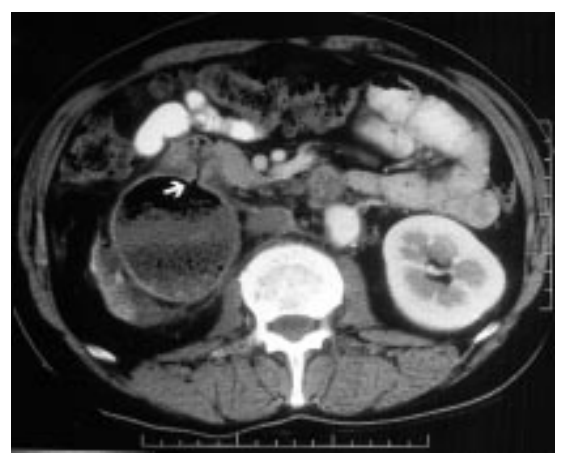

Abb. 2 Parenchymphase: Kontrastmittelanreicherung in der „Zystenwand“. Konturunterbrechung der Raumforderung zum Lumen des Duodenums $(\rightarrow)$. die vom oberen Nierenpol bis an das Duodenum reichte. Eine Verbindung zum Nierenbeckenkelchsystem, das durch die Raumforderung komprimiert und verlagert wurde, ließ sich zunächst nicht nachweisen. Auch zum Duodenallumen war keine Verbindung darzustellen.

In der nativen Computertomographie mit peroraler Kontrastierung des $\mathrm{Ga}$ strointestinaltraktes stellte sich eine $8 \times 8 \mathrm{~cm}$ große zystische Raumforderung dar, die vom oberen Nierendrittel ausging und bis zum Duodenum reichte (Abb.1). In dieser „Zyste“ ließen sich einzelne Gasblasen und Kontrastmittel nachweisen, das zu diesem Zeitpunkt nur aus dem Darm stammen konnte. Nach intravenöser Kontrastmittelapplikation zeigte diese Raumforderung in der Parenchymphase eine Kontrastmittelanreicherung in der „Zystenwand“. In einer Schicht stellte sich eine Verbindung der Raumforderung mit dem Lumen des Duodenums dar (Abb. 2). Die rechte Niere zeigte in diesem Bereich nur einen schmalen Parenchymsaum. Da in der Ausscheidungsphase 4 Minuten nach i.v. KM-Gabe noch kein Übertritt von kontrastiertem Urin in das Lumen der Raumforderung nachweisbar war, wurde eine Spätaufnahme nach 2 Stunden angeschlossen. Hier konnte dann eindeutig ein Kontrastmittelübertritt dokumentiert werden (Abb.3), so daß eine pyeloduodenale Fistel diagnostiziert wurde.

Bei der daraufhin durchgeführten endoskopischen Duodenoskopie zeigte sich postbulbär eine divertikelähnliche gerötete Wandveränderung wie bei Zustand nach einer Perforation. Es wurde zunächst eine perkutane Nierenfistel an-

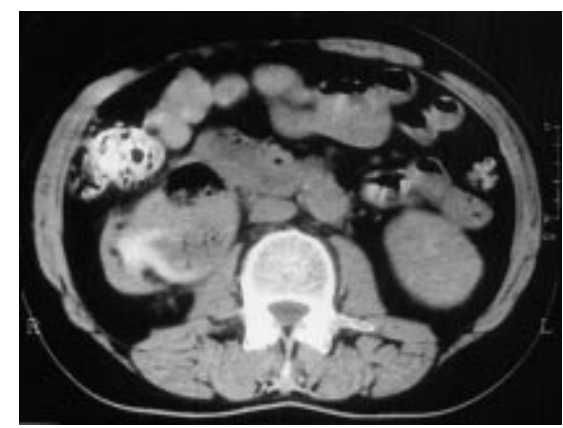

Abb. 3 Spätphase: Übertritt von kontrastiertem Harn in das Lumen der Raumforderung. 
gelegt. Die Analyse des punktierten Sekrets ergab Duodenalflüssigkeit. Intraoperativ bestätigte sich eine große infizierte Hydronephrose mit Einbruch in die Pars descendens des Duodenums. Die rechte Niere wurde entfernt, das Duodenum lokal exzidiert und vernäht. Die histopathologische Untersuchung ergab keinen Hinweis auf eine spezifische Entzündung oder ein malignes Geschehen. Der postoperative Verlauf war komplikationslos.

\section{Diskussion}

Fisteln zwischen dem Harn- und Gastrointestinaltrakt treten in den meisten Fällen im kleinen Becken auf. Hier sind üblicherweise die Harnblase und die benachbarten Darmschlingen betroffen. Eine Verbindung im Bereich des oberen Harntraktes wurde bisher in weniger als 100 Fällen beschrieben. Typischerweise entstehen solche Fisteln zwischen dem Nierenbecken und dem Kolon. Deutlich seltener besteht eine Verbindung zwischen Niere und Duodenum (Culkin et. al., J Urol 1985; 134: 528). Der Ursprung solcher Fisteln kann traumatischer oder spontaner Natur sein. In einer Übersicht von Rodney wurde festgestellt, daß 83\% der Fisteln spontan infolge einer chronischen Entzündung entstanden waren. Interessanterweise traten alle Fälle rechtsseitig auf - wie auch in dem hier vorgestellten Fall (Rodney et al., Urology 1983; 22: 536-539). Neben spezifischen und unspezifischen Entzündungen sind solche Fisteln häufig Folge von Obstruktionen oder Nierenbeckensteinen. Bei der Erosion durch ein Konkrement kommt es zunächst zu einer Urinextravasation, dann zu einem Abszeß und schließlich zu einer inneren Drainage in das Hohlsystem des Intestinums. Die zweithäufigste Ursache sind mit ca. 20\% Traumata, wobei bei indirekten Verletzungen eine vorgeschädigte Niere vorliegen muß (Pulvertaft, Lancet 1935; 1: 24). Direkte Verletzungen kön- nen als Folge von verschluckten Fremdkörpern, Schußverletzungen oder Operationen auftreten (Morris et al., J Urol 1991; 146: 835 -837). Die klinische Symptomatik der Patienten ist unspezifisch. Häufige Symptome sind Fieber, Flankenschmerzen, Übelkeit, Erbrechen, Leukozytose, positive Urinkultur und Diarrhoe. Wegweisend für die Diagnose sind bildgebende Untersuchungen. Während in früheren Arbeiten der Schwerpunkt auf der Kontrastmitteldarstellung lag, steht heute die Sonographie im Vordergrund der Diagnostik (Rodney et al., Urology 1983; 22: 536-539). Wird hier der Verdacht auf eine nephro-enterale Fistel gestellt, sollte man zur Sicherung und präoperativen Planung eine Computertomographie anschließen. In unserem Falle wurde die präoperative Diagnostik noch durch eine perkutane Nierenfistel erweitert.

Bei der Therapie hat sich ein konservatives Vorgehen mit intravenöser Antibiotikagabe nur bei traumatischen Fisteln bewährt. Bei gleichzeitigen Infektionen, chronischen Entzündungen oder Steinen ist die Therapie der Wahl eine Nephrektomie mit gleichzeitigem Verschluß des Darmes über einen retroperitonealen Zugang (Culkin et al., J Urol 1985; 134: 528 und Biondi, J Amer Med Ass 1935; 104: 1894). Hierbei wird eine Kontamination der Peritonealhöhle vermieden.

Nach wie vor sind spontane nephrointestinale Fisteln selten. Durch den großzügigen Einsatz perkutaner Nierenfisteln, die Zunahme laparoskopischer Eingriffe, das vermehrte Wiederauftreten tuberkulöser Infektionen und der anderen oben genannten Risiken sollte man bei entsprechender klinischer Symptomatik in der Differentialdiagnose an eine nephro-intestinale Fistel denken.

T. Hentschel, A. J. Gross, E. Grabbe, Göttingen

\section{Ungewöhnliche Manifestation einer chronisch rekurrierenden multifokalen Osteomyelitis an der Wirbelsäule}

Wirbelkörperdestruktionen beruhen auf verschiedenen demineralisierenden, degenerativen, entzündlichen oder tumorösen Ursachen. Sie können im Einzelfall auch Ausdruck einer chronisch rekur- rierenden multifokalen Osteomyelitis (CRMO) sein, die häufig mit Hauterkrankungen assoziiert ist und als wichtige Komponente des sogenannten SAPHOSyndroms gilt. Der vorliegende Fall zeigt eine seltene Manifestationsform dieser Erkrankung.

\section{Fallbericht}

Eine 25jährige Patientin wurde zur Abklärung von destruktiven Wirbelkörperprozessen stationär aufgenommen. Ein Jahr zuvor waren erstmals rechtsseitige Flankenschmerzen aufgetreten. Die Röntgenuntersuchung der Brust- und Lendenwirbelsäule zeigte seinerzeit einen altersentsprechend unauffälligen Befund. Die Laborparameter waren mit Ausnahme einer wiederholt erhöhten Blutsenkungsgeschwindigkeit bis $40 /$ $70 \mathrm{~mm}$. W. regelrecht. Da in den folgenden 9 Monaten die Beschwerden deutlich zunahmen, wurde die Patientin in die Medizinische Klinik eingewiesen.

Hier bot die Patientin mit Ausnahme eines Klopfschmerzes über der Lendenwirbelsäule einen unauffälligen körperlichen Untersuchungsbefund. Konventionelle radiologische Kontrollaufnahmen der BWS und LWS zeigten nunmehr neben einer links konvexen skoliotischen Fehlhaltung partielle Destruktionen des 12 . BWK sowie des 2 . und 3. LWK (Abb.1). Diese Wirbelkörper wiesen begleitende sklerotische Veränderungen und reaktive Knochenneubildungen auf. Der paravertebrale Weichteilschatten war verbreitert. Die Computertomographie bestätigte ausgedehnte Wirbelkörperdestruktionen mit reaktiven Sklerosierungen und Spicula-ähnlichen Veränderungen, außerdem eine ausgedehnte paravertebrale Weichteilkomponente, die den rechten M.psoas verdrängte und partiell infiltrierte (Abb. 2). Die Magnetresonanztomographie zeigte in den zuvor beschriebenen Regionen pathologische Signalveränderungen und nach intravenöser Kontrastmittelgabe ein deutliches Signalenhancement (Abb. 3a,b). Demgegenüber stellten sich die Bandscheibenfächer völlig unauffällig dar.

Die Befunde wurden insgesamt als Ausdruck einer sog. CRMO bzw. unspezifischen Spondarthritis gedeutet. Passend hierzu zeigte die histologische Untersuchung von Knochenbiopsien eine diskrete lymphoplasmazelluläre Infiltration. Ein Erregernachweis wurde nicht erbracht. Wegen anhaltend erhöhter Entzündungsparameter wurde dennoch eine antibiotische Therapie mit Rifampicin eingeleitet. Ein Kontroll-MRT nach zwei Monaten zeigte ein unverändertes 


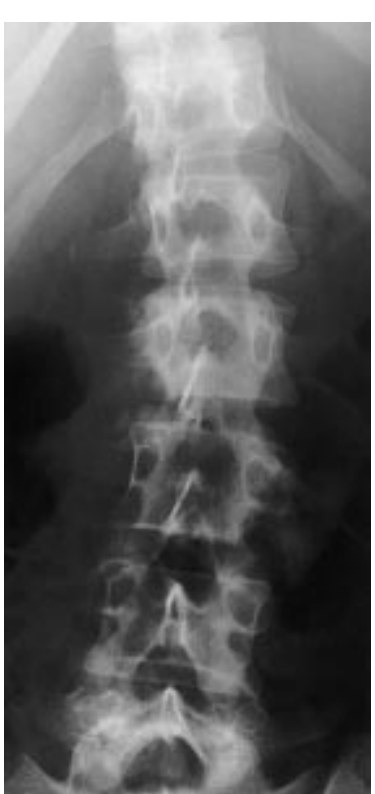

Abb. 1 LWS a.p.: Knöcherne Arrosion des 2. und 3. LWK mit reaktiven sklerotischen Veränderungen. Begleitende Verbreiterung des paravertebralen Weichteilschattens und Ausbildung einer kurzbogigen linkskonvexen Skoliose.

Ausmaß des knöchernen Prozesses und eine leichte Rückbildung der paravertebralen Infiltration.

\section{Diskussion}

Bei der CRMO handelt es sich um eine blande, überwiegend lymphoplasmazelluläre Osteomyelitis, die $\mathrm{zu} \mathrm{90 \%} \mathrm{im}$ Kindesalter auftritt (Schilling F, Fortschr Röntgenstr 1998; 168: 115). Prädilektionsstellen sind die Metaphysen der langen Röhrenknochen und die sternale Hälfte der Clavicula. Seltener ist eine Beteiligung der Wirbelsäule mit multisegmentalem Befall vor allem der unteren BWS (Schilling F, Fortschr Röntgenstr 1998; 168: 115). Die CRMO gilt neben der mit bestimmten Hauterkrankungen assoziierten Arthritis und sterilen Osteitis als ein Hauptkriterium für das sogenannte SAPHO-Syndrom (Kahn MF et al., Baillieres Clin Rheumatol 1994; 8: 333). Dieses Syndrom beinhaltet eine Synovitis, Akne, Pustulose, Hyperostose und Osteitis. Dabei spiegeln die über 50 verschiedenen Bezeichnungen für dieses Syndrom die verwirrenden und z.T. widersprüchlichen Angaben hierzu wider (Boutin RD et al., Amer J Roentgenol 1998; 170: 585). Schilling und Kessler (1998) beschrieben kürzlich die Spondarthritis hyperostotica pustulo-psoria- tica als eine klassische Manifestationsform des SAPHO-Syndroms. Typisch hierfür seien eine Pustulosis palmo-plantaris, eine sternocostoclaviculäre Hyperostose, eine Wirbelsäulenbeteiligung mit Ähnlichkeit zu den in unserem Fall beschriebenen Wirbelkörperprozessen sowie fakultativ eine Extremitätenarthritis. Veränderungen am Achsenskelett bestehen meist in Hyperostosen und Weichteilossifikationen. Diese können unter dem Bild einer bakteriellen Spondylitis oder Osteomyelitis, einer Spondylitis ankylosans oder psoriatica sowie eines DISH-Syndroms verlaufen (Freyschmidt J; Springer 1997. Schilling $\mathrm{F}$ et al., Fortschr Röntgenstr 1998; 169: 576). Dabei gleichen die in einem CT demonstrierten „flammenden Protuberanzen“ an einem Wirbelkörper sehr den Spicula-ähnlichen Veränderungen bei unserer Patientin (Schilling F et al., Fortschr Röntgenstr 1998; 169: 576). Bei ihr lagen vor allem destruktive Wirbelkörperprozesse vor. Diese gelten als eine eher seltene Manifestationsart der Spondylitis hyperostotica pustulo-psoriatica und beziehen dann meist auch den Discus intervertebralis mit ein (Schilling $\mathrm{F}$ et al., Fortschr Röntgenstr 1998; 169: 576). Bei der von uns vorgestellten Patientin ist das Ausmaß der paravertebralen Infiltration sicherlich ungewöhnlich. Außerdem lagen bei ihr im Gegensatz zu vielen Literaturberichten keine Hautveränderungen vor. Bei charakteristischen Knochenläsionen sind diese für die Diagnose eines SAPHO-Syndroms jedoch nicht zwingend erforderlich (Boutin RD et al., Amer J Roentgenol 1998; 170: 585):

Hauterkrankungen können 1. so lange vor den Knochenveränderungen auftreten, daß der Patient sich hieran nicht mehr erinnert, sie können 2. nach den knöchernen Läsionen manifest werden und 3. auch subklinisch verlaufen.

Die Therapie des SAPHO-Syndroms ist üblicherweise symptomatisch. Nach Boutin u. Resnick (1998) beschränkt sie sich meist auf die Gabe von nicht steroidalen Antiphlogistika und ggf. Steroiden. Eine Behandlung mit Antibiotika ist meist erfolglos. Vereinzelt wurde über eine positive Wirkung von alpha-Interferon, Methotrexat, Calcitonin, Biphosphonaten, Vitamin-D-Derivaten und Cyclosporin A berichtet (Boutin RD et al., Amer J Roentgenol 1998; 170: 585). Die Prognose der Erkrankung ist im allgemeinen gut. Nach einem chronisch

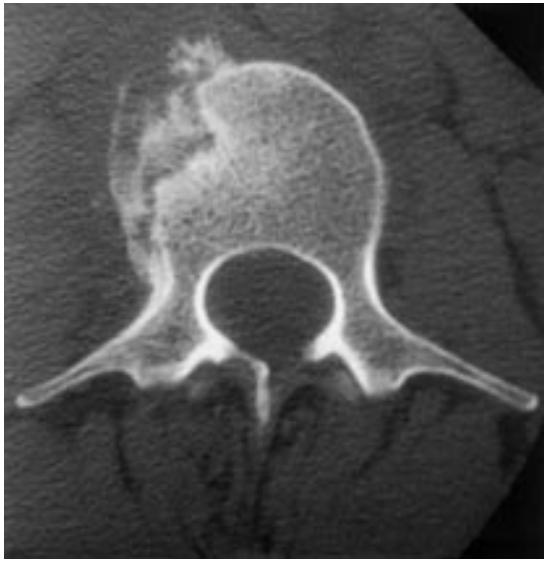

Abb.2 CT des 2.LWK: Destruktion der Wirbelkörpervorderkante mit reaktiven Sklerosierungen, Spicula-ähnlichen Veränderungen und ausgedehnter Weichteilkomponente.

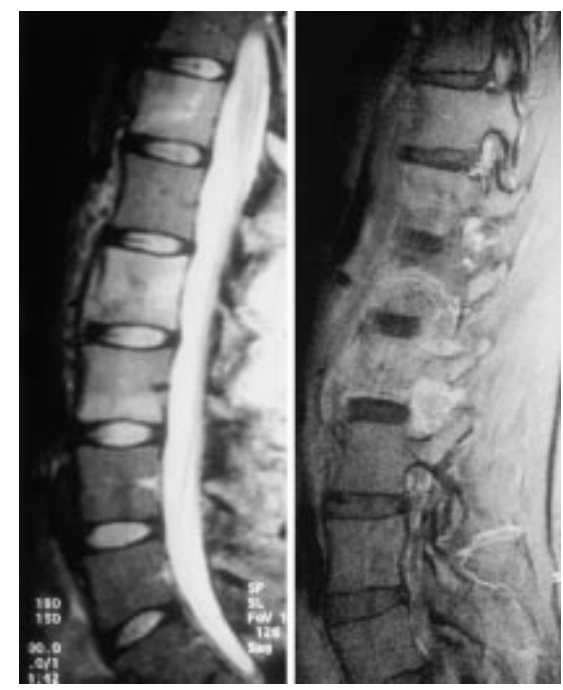

Abb. 3 (a) MRT der LWS, TIRM-Sequenz: Pathologische Signalanhebungen im 12. BWK sowie im 1. und 2. LWK mit partieller Destruktion der Grundplatten und Vorderkanten. (b) MRT der LWS, fettgesättigte $T_{1}$-Sequenz nach intravenöser Kontrastmittelgabe: Ausdehnung der entzündlichen Wirbelkörperprozesse auf den paravertebralen Raum mit Infiltration der Psoasmuskulatur.

rekurrierenden Verlauf über mehrere Jahre kommt es meist zu einer spontanen Heilung (Schilling F, Fortschr Röntgenstr 1998; 168: 115).

C. Funke, E. Grabbe, Göttingen 